\title{
Cranial magnetic resonance imaging in chronic demyelinating polyneuropathy
}

\author{
S H B Hawke, J M Hallinan, J G McLeod
}

nerve biopsy performed in our department and

\begin{abstract}
Twenty one patients with chronic inflammatory demyelinating polyradiculoneuropathy (CIDP) and five patients with chronic demyelinating polyneuropathy associated with benign monoclonal paraproteinaemia none of whom had signs or symptoms of central nervous system disease, had cranial magnetic resonance imaging (MRI) on a 1.5 Tesla unit. Areas of increased white matter signal intensity were seen in one of 10 patients aged less than 50 years and in five of 16 patients aged more than 50 years. In only two of the patients $(8 \%)$, neither of whom had paraproteinaemia, did the appearance strongly suggest demyelination. The only clinical variable that predicted MRI changes was age $(p<$ 0.01).
\end{abstract}

Multiple sclerosis (MS) and chronic inflammatory demyelinating polyradiculoneuropathy (CIDP) are diseases of the central and peripheral nervous systems characterised by chronic multifocal demyelination. Although there are clinical and pathological similarities between MS and CIDP, ${ }^{12}$ it is striking how rarely patients are affected by both diseases. Nevertheless, there are well documented reports of small numbers of patients with clinical and laboratory evidence of both central and peripheral nervous system demyelination. ${ }^{3-5}$ Whether the occurrence of both diseases in the one patient is more than a chance association is uncertain. ${ }^{4}$ If there exists an association between MS and CIDP, a proportion of patients with CIDP might be expected to have evidence of central nervous system demyelination even at a subclinical level and magnetic resonance imaging (MRI) techniques that sensitively detect central nervous system (CNS) demyelination ${ }^{6}$ should identify a higher than expected incidence of white matter changes.

To determine the frequency of MRI white matter signal changes in patients with chronic demyelinating polyneuropathy cranial MRI was performed on 21 patients with CIDP and five patients in whom the chronic demyelinating neuropathy was associated with a benign monoclonal gammopathy. There was no clinical evidence of disease of the CNS in any patient.

\section{Methods}

During 1988, 26 patients who had had sural who satisfied the diagnostic criteria for CIDP, ${ }^{278}$ had cranial MRI on a General Electric Signa 1.5 Tesla unit at the Royal North Shore Hospital in Sydney. Patients were selected on the basis of availability. Sagittal partial saturation images were made with a repetition time (TR) of $600 \mathrm{~ms}$ and an echo-delay time (TE) of $20 \mathrm{~ms}$. Axial spin-echo images were sequenced with a TR of $2600 \mathrm{~ms}$ and a TE of 30 and $80 \mathrm{~ms}$. The images were interpreted blind by a neuroradiologist $(\mathrm{JH})$ and assessed for white matter signal changes. Areas of increased signal were not regarded as significant if they were: $<2 \mathrm{~mm}$ in largest diameter, considered to be normal patterns or degraded by artifact. Attention was given to the distribution of white matter signal changes.

All patients had been examined by one of us and were being regularly reviewed. No patient had clinical or other evidence of central nervous system dysfunction. CIDP was classified as relapsing or non-relapsing. ${ }^{2}$ Active disease was defined as relapse within the 12 months before MRI or dependency on immunosuppressive treatment or plasma exchange at the time of MRI. A disability grading scale ${ }^{8}$ was used to assess functional disability at the time of MRI.

To investigate the possibility of certain clinical variables predicting abnormal MRI the following statistical methods were used where appropriate: Student's $t$ test; Chi-square analysis; and the Wilcoxon rank sum test.

\section{Results}

Clinical features (table 1)

There were 21 patients with CIDP ( 17 male, four female). The mean age at onset was 42 years (range 16-74; SD 13 years). The mean age at the time of MRI was 52 years (range 34 78; SD 11 years). Twelve patients had relapsing disease and nine had a non-relapsing course. The disease was considered active using the criteria described above in eight patients and inactive in 13 patients. Most patients had mild to moderate disability when MRI was performed.

There were five patients in whom the demyelinating neuropathy was associated with benign monoclonal gammopathy (IgGk = two patients; $\operatorname{Ig} M \lambda=$ three patients). The clinical characteristics were similar to those without paraproteinaemia. The mean age at onset was 57 years (range 23-70; SD 21) years. The mean age at the time of MRI was 65 years (range 3379; SD 19 years). Three patients had a relapsing and two patients a non-relapsing course. All patients had active disease at the time of MRI. 
Table Clinical features and results of MRI in patients with chronic demyelinating neuropathy

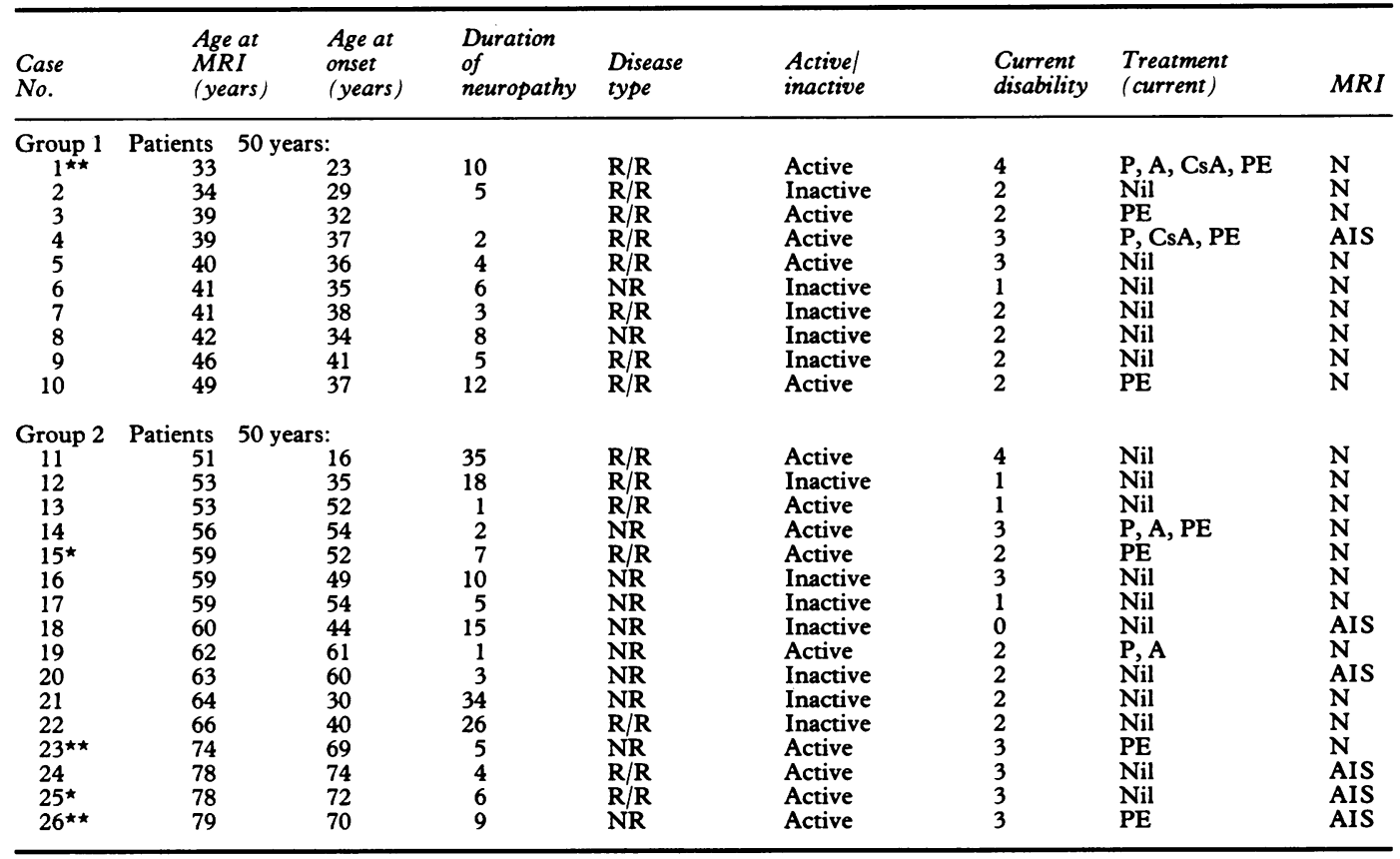

R/R Relapsing/remitting or relapsing progressive disease; NR, non-relapsing disease; *patients with IgG kappa paraproteinaemia; ${ }^{\star \star}$ patients with IgM lambda paraproteinaemia.

Treatment: $P$, prednisone; A, azathioprine; CsA, cyclosporine A; PE, plasma exchange.

Disability scale (8): 0 , normal; 1 , neurological signs but no symptoms or vice-versa; 2 , mild motor and/or sensory symptoms with signs; 3, moderately disabled by motor and sensory symptoms, including ataxia; 4, requiring assistance with eating and dressing, or using a walking aid; 5 , not ambulant.

MRI: N, no significant white matter changes; AIS, areas of increased signal intensity in white matter.

\section{Magnetic resonance imaging}

Since there was no difference in the findings in the patients with and without a paraproteinaemia the results were considered together (table 1). Six patients $(24 \%)$ had areas of increased white matter signal intensity by our criteria. For the purposes of interpreting the MRI results it was useful to consider two groups of subjects: patients $<50$ years (group 1); patients $>50$ years (group 2). ${ }^{9}$ Of the 10 patients in group 1 , only one patient aged 39 years without paraproteinaemia (case 4) had an abnormal white matter signal, a single area 6$10 \mathrm{~mm}$ in diameter adjacent to the right trigone (fig 1).

Five of 16 patients in group two had areas of increased white matter signal intensity. Two of

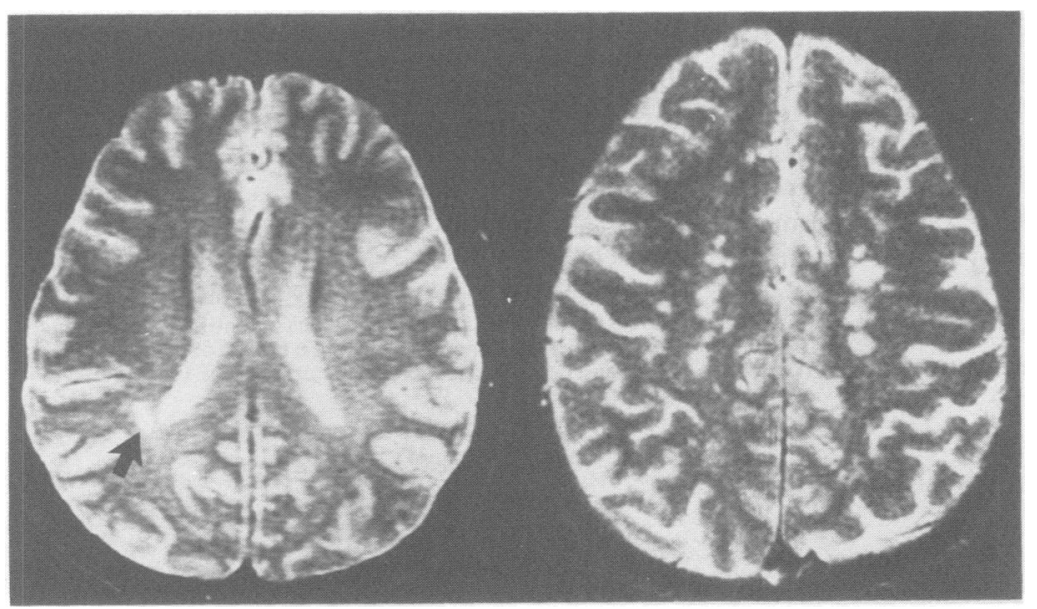

Figure T2 weighted axial image of a patient aged 39 years (case 4) showing a single area of increased white matter signal adjacent to the right trigone (left), and of a patient aged 63 years (case 20) showing areas of increased white matter signal in the periventricular regions of both cerebral hemispheres (right). them (cases 18 and 26) had multiple small $(<5 \mathrm{~mm})$ rounded densities scattered uniformly throughout the white matter of both cerebral hemispheres consistent with subcortical small vessel disease. One patient without paraproteinaemia (case 20) with multiple areas of paraventricular increased density had changes that suggested demyelination (fig 1). In the others (cases 24 and 25) the scan appearances were non-specific. An incidental venous angioma was noted in one patient.

The only clinical variable that correlated with an abnornal scan was age at the time of MRI ( $p<0.01)$. There was no correlation between an abnormal MRI and the following: sex, the duration of disease before scanning, current activity of CIDP, pattern of disease, current patient disability, nor current treatment status.

\section{Discussion}

All patients in the study had chronic demyelinating polyneuropathy, confirmed by sural nerve biopsy. Twenty one patients satisfied the accepted criteria for CIDP ${ }^{27}$ most of whom had been included in a previous study of 92 patients. ${ }^{2}$ They were representative of the larger group in respect of age, sex, clincal features, proportion of relapsing and nonrelapsing cases, and disability. An additional five cases had similar clinical electrophysiological and pathological features but also had an associated monoclonal paraproteinaemia. Whether these cases of chronic demyelinating neuropathy with benign paraproteinaemia are variants of CIDP remains uncertain.

None of the patients had signs or symptoms 
of CNS dysfunction. Areas of increased signal intensity were demonstrated on T2 and intermediate-weighted MRI in five of 16 patients $(31 \%)$ over the age of 50 years, but in only one of 10 patients $(10 \%)$ aged less than 50 years.

Although there were no age-matched controls included in our study, there are now data available in the published literature that clearly show an increased frequency of white matter signal abnormalities with increasing age in healthy subjects. ${ }^{10-12}$

In a study using a 1.5 Tesla magnet, Fazekas et $a l^{12}$ found parenchymal white matter signal changes in 27 of 44 healthy volunteers free of cerebrovascular symptoms. Awad et al ${ }^{13}$ found that incidental white matter signal changes seen at necropsy MRI in seven elderly patients dying of non-neurological disease were due to sub-cortical atherosclerosis. It is likely therefore that white matter changes visualised in elderly patients are the results of vascular disease rather than of primary demyelination.

Criteria have recently been suggested in order to increase the specificity of MRI in demyelinating disease in older age groups. Using these criteria for primary demyelination (that is, two or more of the following findings: (a) lesion size $>6 \mathrm{~mm}$; (b) lesions abutting the bodies of the lateral ventricles; and $(c)$ infratentorial white matter lesions), only one of the patients over 50 years old in this study would have had a scan indicating demyelination (case 20 , who fulfilled criteria $a$ and $b$ ).

In the group of younger patients, in which the most conclusive information to support the existence of concurrent central and peripheral nerve demyelination is likely to be found, MRI was abnormal in only one patient in whom a single area of increased white matter signal was imaged. In a series of younger control subjects abnormalities have been found in small numbers. ${ }^{14}$ Although some authors have viewed the occurrence of white matter changes in younger healthy subjects as indicative of sub-clinical demyelination ${ }^{6}$ there is no pathological data to support this contention and thus the nature of these lesions is unproven.

Thomas et $a l^{4}$ reported six cases in which a chronic demyelinating neuropathy was associated with a relapsing multifocal CNS disorder resembling multiple sclerosis. In the five patients in whom it was performed, MRI showed lesions indistinguishable from those observed in MS. Rubin et $\mathrm{al}^{3}$ also reported two patients with CIDP with clinical evidence of CNS demyelination. In the patient in whom it was performed, MRI demonstrated multiple periventricular and brainstem abnormalities. Mendell et $a l^{5}$ reported MRI findings characteristic of CNS demyelination in three patients with CIDP associated with clinical evidence of CNS involvement. Three other patients had abnormal MRI but no clinical evidence of CNS disease. These three patients were all aged 70 years or more and the abnormalities found may be demonstrated in healthy subjects of the same age.

Although there are similarities between CIDP and $M S,{ }^{12}$ and in some patients peripheral and central nervous system demyelination may occur concurrently, in this study changes strongly suggestive of CNS demyelination were demonstrated in only two of 26 patients by MRI and in none by clinical criteria. Moreover, there was no clinical evidence of CNS demyelination in a previously reported large series of patients with CIDP. ${ }^{2}$

Dr W A Sorby, Magnetic Resonance Imaging Unit, Royal North Shore Hospital, was responsible for the MRI on the patients in the study. We are grateful for his help and cooperation.

1 Prineas JW. Demyelination and remyelination in recurrent idiopathic polyneuropathy. An electron microscope study. Acta Neuropathol (Berl) 1971;18:34-57.

2 McCombe PA, Pollard JD, McLeod JG. Chronic inflammatory demyelinating polyradiculoneuropathy. A clinical matory demyelinating polyradiculoneuropathy. A clinical 110:1617-30.

3 Rubin M, Karpati G, Carpenter S. Combined central and peripheral myelinopathy. Neurology 1987;37:1287-90.

4 Thomas PK, Walker RWH, Rudge P, et al. Chronic demyelinating peripheral neuropathy associated with multifocal central nervous system demyelination. Brain 1987;110:53-76.

5 Mendell JR, Kolkin S, Kissel JT, Weiss KL, Chakeres DW, Rammohan KW. Evidence for central nervous system demyelination in chronic inflammatory demyelinating polyradiculoneuropathy. Neurology 1987;37:1291-4.

6 Ormerod IEC, Miller DH, McDonald WI, et al. The role of NMR imaging in the assessment of multiple sclerosis and isolated neurological lesions. Brain 1987;110:1579-616.

7 Dyck PJ, Lais AC, Ohta M, Bastron JA, Okazaki H, Groover Dyck PJ, Lais AC, Ohta M, Bastron JA, Okazaki H, Groover Mayo Clinic Proc 1975;50:621-37.

8 Prineas JW, McLeod JG. Chronic relapsing polyneuritis. J Neurol Sci 1976;27:427-58.

9 Fazekas F, Offenbacher H, Fuchs S, et al. Criteria for an increased specificity of MRI interpretation in elderly subjects with suspected multiple sclerosis. Neurology 1988;38:1822-5.

10 Bradley WG, Waluch V, Brant-Zawadzki M, Yadley RA Wycoff $R$. Patchy periventricular white matter lesions in the elderly: common observations during NMR imaging. Non Invasive Med Imaging 1984;1:35-41.

11 Brant-Zawadzki M, Fein G, Van Dycke C, Kiernan R, Davenport L, de Groot J. MR Imaging of the aging brain: Patchy white-matter lesions and dementia. AJNR 1985;6: 675-82.

12 Fazekas F, Niederkorn $\mathrm{K}$, Schmidt $\mathrm{R}$, et al. White matter signal abnormalities in normal individuals: Correlation signal abnormalities in normal individuals: Correlation with carotid ultrasonography cerebral blood flow measurements, and

13 Awad IA, Johnson PC, Spetzler RF, Hodak JA. Incidental subcortical lesions identified on magnetic resonance imaging in the elderly. II. Postmortem correlations. Strok 1986;17:1090-7.

14 Awad IA, Spetzler RF, Hodak JA, Awad CA, Carey R. Incidental subcortical lesions identified on magnetic resonance imaging in the elderly. I. Correlation with age and cerebrovascular risk factors. Stroke 1986;17:1084-9. 